\title{
Induction of HLA-A*33-restricted cytotoxic lymphocytes against renal cell carcinoma targeting galectin 9 and PINCH
}

\author{
HIDENORI KAWASHIMA $^{1,2}$, SAKAE MASAKI $^{2}$ and MARIE KAWAMURA ${ }^{2}$ \\ ${ }^{1}$ Shirahama Hamayu Hospital, Shirahama, Wakayama 649-2211; ${ }^{2}$ Department of Urology, \\ Osaka City University Graduate School of Medicine, Osaka 545-8585, Japan
}

Received July 10, 2014; Accepted August 1, 2014

DOI: $10.3892 /$ br.2014.334

\begin{abstract}
Galectin 9, a ligand of T cell immunoglobulin and mucin domain 3 (TIM-3), and PINCH, an epithelial-to-mesenchymal transition (EMT)-promoting molecule, are expressed at much higher levels in cancerous lesions of clear cell type renal cell carcinoma ( $\mathrm{RCC}$ ) compared to normal renal tissues, and their expression levels are extremely low in normal tissues, except for galectin 9 in the spleen. Galectin 9- and PINCH-derived peptides have previously been shown to induce human leukocyte antigen (HLA)-A*2402-restricted and HLA-A*0201-restricted cytotoxic lymphocytes (CTLs) with specific and highly cytotoxic activities toward RCC cells. The present study aimed to identify the peptides that induced HLA-A*33-restricted CTLs that exhibited specific and highly cytotoxic activities toward RCC cells. Specific CTLs were induced significantly, as shown by cluster of differentiation 107a degranulation stimulated with VMRC-RCW renal carcinoma cells. Therefore, peptide vaccines targeting galectin 9 and PINCH appear to be promising for clinical application.
\end{abstract}

\section{Introduction}

Thus far, the main drugs for treating metastatic renal cell carcinoma (RCC) patients are molecular targeting drugs, including tyrosine kinase and mammalian target of rapamycin inhibitors. However, immunotherapy using interferon has been shown to be extremely effective at achieving complete remission (CR), although the response rate was as low as 15.9 and $1.8 \%$ for partial remission (PR) and CR, respectively (1). A Japanese multi-center study reported that RCC patients with lung metastases exhibited a survival benefit from cytokine therapies (2). Thus, it is believed that RCC-specific antigens

Correspondence to: Dr Hidenori Kawashima, Shirahama Hamayu Hospital, 1447, Shirahama, Nishimuro, Wakayama 649-2211, Japan

E-mail: kawashima@hamayu-hp.or.jp

Key words: galectin 9, immune checkpoints, $\mathrm{PINCH}$, renal cell carcinoma, epithelial-to-mesenchymal transition, cytotoxic T lymphocytes exist and that cytokine therapy causes clear responses at times when specific anti-tumor immunity is acquired.

By screening an expression library of RCC with the sera of cytokine therapy responders, two antigens were identified, galectin 9 and $\mathrm{PINCH}$, which reacted with the sera of patients with metastatic RCC that responded extremely well to cytokine therapy. Our previous study considered the ability of cytokine therapy responders to acquire specific cancer immunity with specific antibodies (3). Galectin 9 is a ligand of one of the immune checkpoint molecules, T cell immunoglobulin and mucin domain 3 (TIM-3), that suppresses T-cell activity (4) and PINCH is an epithelial-to-mesenchymal transition (EMT)-promoting molecule (5) that contributes to apoptosis resistance in cancer cells (6). These two antigens appear to promote cancer cell survival and thus are good immunotherapy targets. Using peptides derived from these antigens, our previous study induced human leukocyte antigen (HLA)-A*2402-restricted and HLA-A*0201-restricted cytotoxic lymphocytes (CTLs) with specific and highly cytotoxic activities toward RCC cells, which indicates that immunotherapy targeting these antigens is promising (3).

Together with HLA-A*2402 and HLA-A*0201, HLA-A*33 is a significant HLA type present in $<20 \%$ of Japanese. The aim of the present study was to identify the peptides that abundantly induced HLA-A*33-restricted CTLs that exhibited specific and highly cytotoxic activities toward RCC cells.

\section{Materials and methods}

Materials. The kidney cancer cell lines, VMRC-RCW and TUHR-14TKB; the pancreas cancer cell line, PK-45P; and the testicular cancer cell line, NEC8; were purchased from Riken Bioresource Center (Riken BRC; Tsukuba, Japan). The urothelial cancer cell line, UM-UC-3, was from American Type Culture Collection (Manassas, VA, USA). A human B lymphocyte cell line transformed with Epstein-Barr virus, RBRC-HEV0091 (HLA-A*0201/A*3303), was obtained from Riken BRC. Chromium-51 radionuclide $\left({ }^{51} \mathrm{Cr}\right)$ was purchased from PerkinElmer Japan Co., Ltd. (Yokohama, Japan). Takara Taq DNA polymerase was purchased from Takara Bio, Inc. (Otsu, Japan). Ficoll-Paque Plus was purchased from GE Healthcare Bio-Sciences (Uppsala, Sweden). Allophycocyanin (APC) mouse anti-human cluster of differentiation 8 (CD8), fluorescein isothiocyanate (FITC) mouse 
anti-human CD107a and the Human CD8 T-Lymphocyte Enrichment set were from BD Sciences (Franklin Lakes, NJ, USA).

Reverse transcription-polymerase chain reaction (RT-PCR). Total RNA was extracted from cultured cells using RNAqueous $^{\mathrm{TM}}$ purchased from Ambion, Inc. (Austin, TX, USA) and $0.2 \mu \mathrm{g}$ of total RNA was used as a template for RT-PCR. The set of primers used for galectin 9 were F: 5'-ATG GCCTTCAGCGGTTCCCAG-3' and R: 5'-CTATGTCTGCAC ATGGGTCAG-3'; and for PINCH were F: 5'-AAGATAATT CGCAGTGATGTGAA-3' and R: 5'-GTAGATCAAGACAAG TAATGTTG-3'. PCR for galectin 9 was performed using the following temperature conditions: Denaturing, $94^{\circ} \mathrm{C}$; annealing, $65^{\circ} \mathrm{C}$; and polymerization, $72^{\circ} \mathrm{C}$. For $P I N C H$, the temperature for annealing was $55^{\circ} \mathrm{C}$. The PCR reactions for galectin 9 and $P I N C H$ included 30 and 33 cycles, respectively. Glyceraldehyde 3-phosphate dehydrogenase $(G 3 P D H)$ expression was used as an internal control.

Determination of HLA typing. HLA typing of volunteer blood and cell lines was analyzed and determined by SRL, Inc. (Tachikawa, Japan) using the PCR-sequencing based typing method.

CTL induction with peptides. The amino acid sequences of the candidate peptides were determined using the 'Bioinformatics and Molecular Analysis Section (BIMAS; National Institutes of Health) software for HLA peptide binding predictions' (BIMAS, Bethesda, MD, USA). The galectin 9-derived peptide, DIAFHFNPR, was designated as $9 \mathrm{~m} 57$ and the PINCH-derived peptide, DIGFVKNAGR, was designated as $10 \mathrm{~m} 106$. Peripheral blood mononuclear cells (PBMCs) $\left(3 \times 10^{6}\right.$ cells/well) from HLA-A*3303 healthy donors were isolated using Ficoll-Paque Plus and cultured with RPMI-1640 containing $10 \%$ human $\mathrm{AB}$ type-serum, $50 \mathrm{U} / \mathrm{ml}$ of penicillin, $50 \mu \mathrm{g} / \mathrm{ml}$ of streptomycin and $5 \mu \mathrm{M}$ of the peptide in a 24-well plate. One week later, stimulator cells (that is antigen presenting cells; APCs) of the same HLA type (RBRC-HEV0091) were pulsed with the peptides at $3 \mu \mathrm{M}$ and treated with irradiation (80 Gy), and the PBMCs were stimulated by co-culturing with the APCs at a responder/stimulator ratio of 5:1. Two days after stimulation, $20 \mathrm{IU} / \mathrm{ml}$ interleukin-2 (IL-2) was added. Subsequently, half of the medium was changed with new medium containing $40 \mathrm{IU} / \mathrm{ml}$ IL-2 every two days. Stimulation with the peptide-pulsed APCs was repeated every 10 days for a total of 4-5 times. At the final stimulation, $\mathrm{CD}^{+}$cells were isolated using the Human CD8 T-Lymphocyte Enrichment set and stimulated with the peptides-pulsed APC. Five to six days later, the $\mathrm{CD} 8{ }^{+}$cells were subjected to the ${ }^{51} \mathrm{Cr}$-releasing assay.

${ }^{5 l}$ Cr-releasing assay. Target cells $\left(1 \times 10^{6}\right.$ cells), including VMRC-RCW, TUHR-14TKB, PK-45P, NEC8 and UM-UC-3, were labeled with $50 \mu \mathrm{Ci}{ }^{51} \mathrm{Cr}$ for $1.5 \mathrm{~h}$ at $37^{\circ} \mathrm{C}$ and washed with RPMI-1640 three times. Subsequently, $2 \times 10^{4}{ }^{51} \mathrm{Cr}$-labeled target cells were cultured with effector cells at effector cell:target cell (E:T) ratios of 1:1, 3:1 and $10: 1$ at $37^{\circ} \mathrm{C}$ for $4 \mathrm{~h}$ in a U-shape bottom 96 -well plate. Following incubation, the plate was centrifuged at $400 \mathrm{x}$ g for $5 \mathrm{~min}$ and the supernatants were subjected to measurement with a $\gamma$-counter. The assays

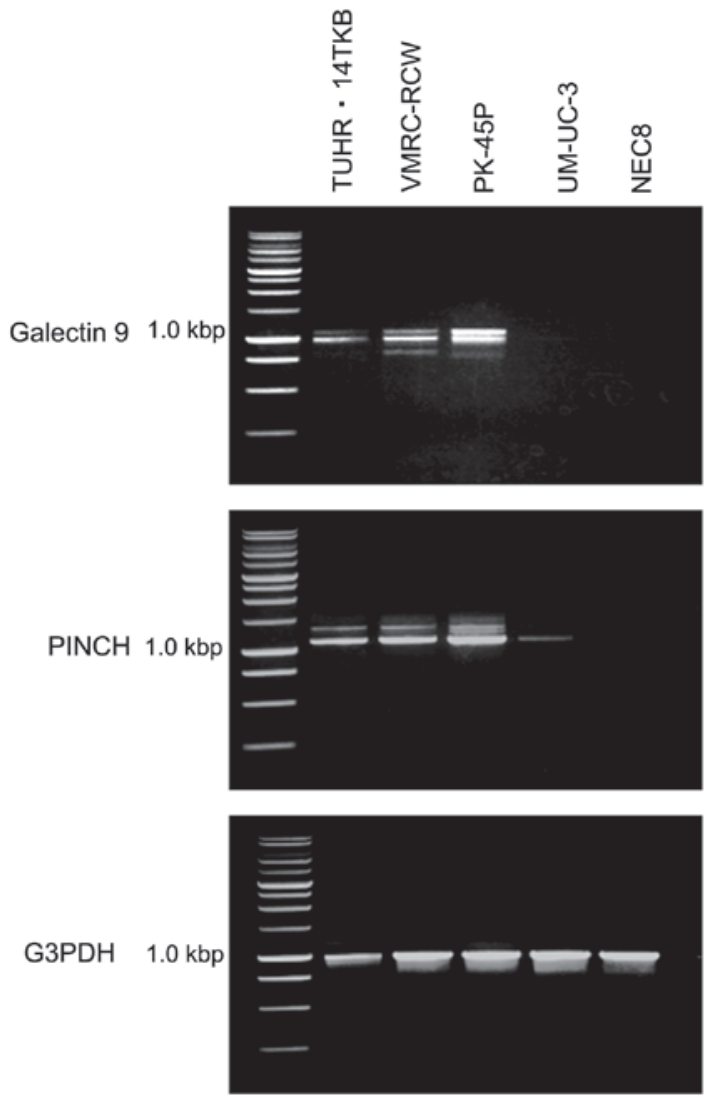

Figure 1. RNA expression of galectin 9 and $P I N C H$ in cancer cell lines used for the cytotoxicity assay. Reverse transcription-polymerase chain reaction analysis of galectin 9 and $P I N C H$ in various cancer cell lines, including renal cell carcinoma cell lines, TUHR-14TKB and VMRC-RCW; the pancreas cancer cell line, PK-45P; the urothelial cancer cell line, UM-UC-3; and the testicular cancer cell line, NEC8. G3PDH, glyceraldehyde 3-phosphate dehydrogenase.

were performed in triplicate and the values represent the mean \pm standard error.

CD107a staining and flow cytometry analysis. CTLs $\left(3 \times 10^{5}\right.$ cells) were incubated with VMRC-RCW target cells or NEC8 control cells at a ratio of $3: 1$ for $2.5 \mathrm{~h}$ at $37^{\circ} \mathrm{C}$ in medium containing FITC-conjugated anti-CD107a antibody. The cells were subsequently labeled with APC-conjugated anti-CD8 antibody, washed and analyzed using a BD LSR II flow cytometer (BD Sciences).

\section{Results}

RNA expression of galectin 9 and PINCH in cancer cell lines. Galectin 9 was expressed in the renal cancer cell lines, TUHR-14TKB and VMRC-RCW, and the pancreas cancer cell line, PK-45P. No expression was detected in the other cancer cell lines, such as UM-UC-3 and NEC8. PINCH was expressed in TUHR-14TKB, VMRC-RCW, PK-45P and UM-UC-3 cells, whereas it was not expressed in the testicular cancer cell line, NEC8 (Fig. 1).

HLA-A*33-restricted CTLs induced by peptides to galectin 9 and PINCH exhibit antigen-specific and highly cytotoxic activities toward the VMRC-RCW RCC cell line. The amino 
A \%specific lysis

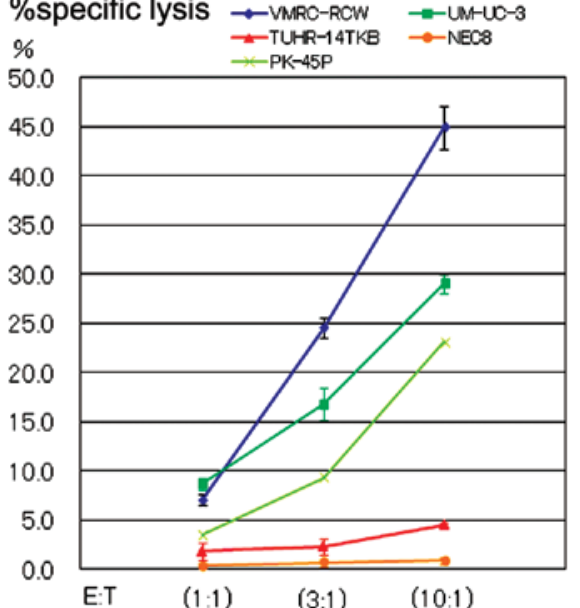

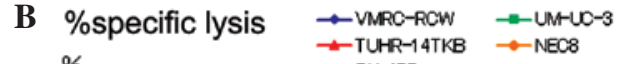

$\% \rightarrow$ TUHR-14TKB $\rightarrow$ NEC8

70.0

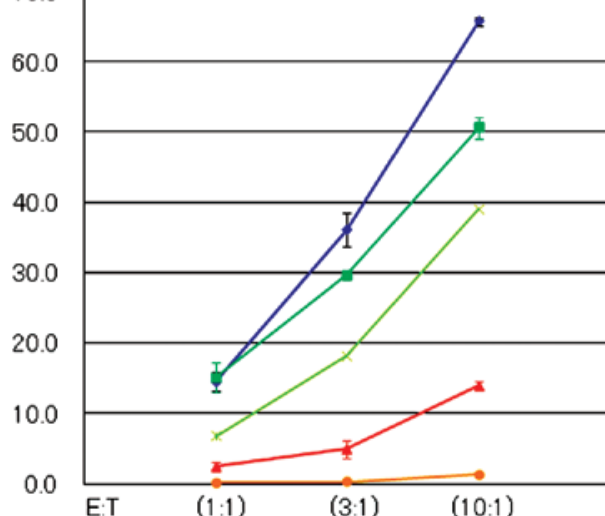

VMRC-RCW: $A * 3303$, galectin $9(+)$. PINCH(+); UM-UC3: A*3301, galectin $9(-)$, PINCH (+); TUHR-14TKB: A*2402, galectin $9(+), \mathrm{PINCH}(+)$; NEC8: $A * 2402$, galectin $9(-)$, $\mathrm{PINCH}(-)$. PK45-P: A*3303, galectin 9(+), $\mathrm{PINCH}(+)$;

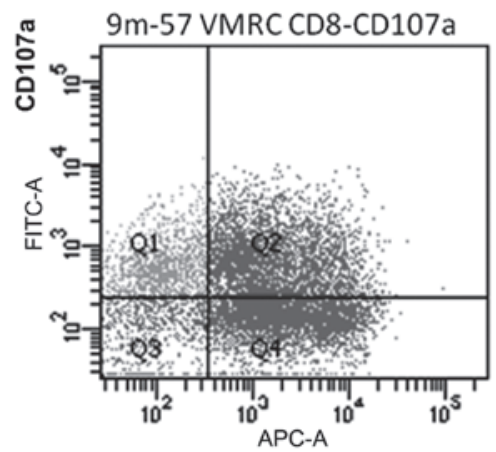

D

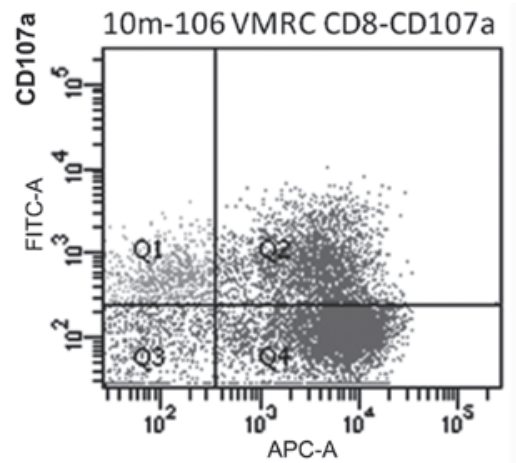

Figure 2. Antigen-specific and human leukocyte antigen (HLA)-A*33-restricted cytotoxicity of cytotoxic lymphocytes (CTLs) induced by the galectin 9 and PINCH peptides. (A) CTLs were induced using peripheral blood mononuclear cells from healthy volunteers (HLA-A*3303) with the galectin 9-derived peptide, designated as $9 \mathrm{~m} 57$. The cytotoxicity of the CTLs toward several cancer cell lines, including the renal cell carcinoma line, VMRC-RCW, was assessed by the chromium-51 radionuclide $\left({ }^{51} \mathrm{Cr}\right.$ )-releasing assay. (B) The cytotoxicity of CTLs induced with the PINCH-derived peptide, designated as $10 \mathrm{~m} 106$, was measured by the ${ }^{51} \mathrm{Cr}$-releasing assay. (C and D) Cluster of differentiation 107a (CD107a) degranulation was observed by co-culturing with VMRC-RCW cells CTLs induced with (C) $9 \mathrm{~m} 57$ or (D) $10 \mathrm{~m} 106$ were stimulated with VMRC-RCW cells in medium containing fluorescein isothiocyanate (FITC)-conjugated anti-CD107a antibody and subsequently labeled with allophycocyanin (APC)-conjugated anti-CD8 antibody, followed by flow cytometry analysis.

acid sequences of the candidate peptides for CTL induction were determined using a database named the 'BIMAS software for HLA peptide binding predictions'.

Antigen-specific and HLA-A*33-restricted cytotoxicities of the CTLs induced by the galectin 9-derived peptide, DIAFHFNPR, designated as 9m57 and the PINCH-derived peptide, DIGFVKNAGR, designated as $10 \mathrm{~m} 106$, are shown in Fig. 2A and B, respectively. The CTLs induced by the peptides $9 \mathrm{~m} 57$ and $10 \mathrm{~m} 106$ showed cytotoxicity toward the renal cancer cell line VMRC-RCW (HLA-A*3303+, galectin $9^{+}$and $\mathrm{PINCH}^{+}$) with high activities at the E:T ratio of 10:1. The CTLs were not active toward the cancer cells with different HLA-A or negative antigens. However, the CTLs induced by the galectin 9-derived peptide were active toward UM-UC-3 cells

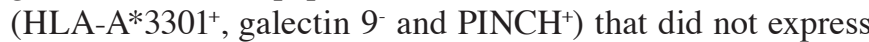
galectin 9, as determined by RT-PCR.

CD107a degranulation was observed by co-culturing with VMRC-RCW cells in CD8 ${ }^{+} \mathrm{T}$ cells (CTLs) that were induced by the peptides for galectin $9(9 \mathrm{~m} 57)$ and PINCH $(10 \mathrm{~m} 106)$ (Fig. 2C and D). Co-incubation with the control NEC8 cells did not cause CD107a degranulation.

\section{Discussion}

RCC has been considered to be immunogenic, as in extremely rare cases metastatic lesions spontaneously regress following the removal of the primarily involved site, and the phenomenon is presumed to be a result of an immune response. Thus, prior to the present era of molecular targeting drugs, cytokine therapy had been widely chosen and demonstrated to achieve results, although the response rates were low $(1,2)$.

Peptide vaccines that are aimed for specific immunotherapy showed limited clinical effects on metastatic RCC (7-12). One possible explanation is that the targeting tumor antigens used were not capable of inducing strong CTLs, which possibly results in the existence of other antigens playing more central roles in cancer immunity.

Our previous study screened an expression library of RCC using the sera of cytokine therapy responders as a probe, as it was considered that those responders acquired specific and strong immunity against RCC. Through this screening, two novel antigens of RCC were identified: Galectin 9 and PINCH (3). Galectin 9 is a ligand of the immune checkpoint 
protein TIM-3 that suppresses T-cell activity (4) and PINCH is an EMT-promoting molecule (5) that contributes to apoptosis resistance in cancer cells (6). Functionally, these two antigens appear to be good immunotherapy targets. Peptides derived from these antigens induced CTLs of HLA-A*2402 and HLA-A*0201, which showed highly cytotoxic activities toward RCC cells of the corresponding HLA-A (3).

Recently it was demonstrated that cancer cells exploit immune checkpoint molecules that mediate suppressing signals of immunity to escape from the immune surveillance system, and drugs (antibodies) targeting these checkpoints molecules, including CTL-associated protein 4 (CTLA-4), programmed cell death protein 1 (PD-1) and programmed death-ligand 1 (PD-L1), have been developed (13). In RCC, clinical studies on nivolumab (BMS-936558), an anti-PD-1 antibody $(14,15)$, and MPDL-3280A, a humanized monoclonal antibody against PD-L1 (15), have been reported. Among these immune checkpoint inhibitors, nivolumab achieved $27 \%$ of the response rates $(\mathrm{PR}+\mathrm{CR})$ in treating RCC (14).

Increasing attention has been paid to TIM-3 as a third immune checkpoint receptor following PD-1 and CTLA-4. TIM-3 binds to its ligand, galectin 9, resulting in T-cell suppression (4). Combination therapy of anti-TIM-3 antibody with anti-CTLA-4 and anti-PD-1 antibodies showed clear and additive efficacy in animal experiment models (16). In the present study, overexpression of galectin 9 in RCC is notable as CTLs induced by galectin 9-derived peptides attack RCC and also remove the immunosuppressive control by immune checkpoints.

Together, the peptide vaccines targeting galectin 9 and PINCH appear to be promising and further studies examining their clinical use are necessary.

\section{Acknowledgements}

Financial support was provided by 'Chizai Katsuyo Sokushin Highway' from the Japan Science and Technology Agency and grants from the Osaka City University Medical Research Foundation.

\section{References}

1. Umeda T and Niijima T: Phase II study of alpha interferon on renal cell carcinoma. Summary of three collaborative trials. Cancer 58: 1231-1235, 1986.
2. Naito S, Yamamoto N, Takayama T, Muramoto M, Shinohara N, Nishiyama K, Takahashi A, Maruyama R, Saika T, Hoshi S, et al: Prognosis of Japanese metastatic renal cell carcinoma patients in the cytokine era: a cooperative group report of 1463 patients. Eur Urol 57: 317-325, 2010.

3. Kawashima H, Obayashi A, Kawamura M, Masaki S, Tamada S, Iguchi T, Uchida J, Kuratsukuri K, Tanaka T and Nakatani T: Galectin 9 and PINCH, novel immunotherapy targets of renal cell carcinoma: a rationale to find potential tumor antigens and the resulting cytotoxic $\mathrm{T}$ lymphocytes induced by the derived peptides. BJU Int 113: 320-332, 2014.

4. Zhu C, Anderson AC, Schubart A, Xiong H, Imitola J, Khoury SJ, Zheng XX, Strom TB and Kuchroo VK: The Tim-3 ligand galectin-9 negatively regulates $\mathrm{T}$ helper type 1 immunity. Nat Immunol 6: 1245-1252, 2005.

5. Li Y, Dai C, Wu C and Liu Y: PINCH-1 promotes tubular epithelial-to-mesenchymal transition by interacting with integrin-linked kinase. J Am Soc Nephrol 18: 2534-2543, 2007.

6. Chen K, Tu Y, Zhang Y, Blair HC, Zhang L and Wu C: PINCH-1 regulates the ERK-Bim pathway and contributes to apoptosis resistance in cancer cells. J Biol Chem 283: 2508-2517, 2008.

7. Van Poppel H, Joniau S and Van Gool SW: Vaccine therapy in patients with renal cell carcinoma. Eur Urol 55: 1333-1342, 2009.

8. Uemura H, Fujimoto K, Tanaka M, Yoshikawa M, Hirao Y, Uejima S, Yoshikawa K and Itoh K: A phase I trial of vaccination of CA9-derived peptides for HLA-A24-positive patients with cytokine-refractory metastatic renal cell carcinoma. Clin Cancer Res 12: 1768-1775, 2006.

9. Rahma OE, Ashtar E, Ibrahim R, Toubaji A, Gause B, Herrin VE, Linehan WM, Steinberg SM, Grollman F, Grimes G, et al: A pilot clinical trial testing mutant von Hippel-Lindau peptide as a novel immune therapy in metastatic renal cell carcinoma. J Transl Med 8: 8, 2010.

10. Iiyama T, Udaka K, Takeda S, Takeuchi T, Adachi YC, Ohtsuki Y, Tsuboi A, Nakatsuka S, Elisseeva OA, Oji Y, et al: WT1 (Wilms' tumor 1) peptide immunotherapy for renal cell carcinoma. Microbiol Immunol 51: 519-530, 2007.

11. Sato E, Torigoe T, Hirohashi Y, Kitamura H, Tanaka T, Honma I, Asanuma H,Harada K, Takasu H, Masumori N, et al: Identification of an immunogenic CTL epitope of HIFPH3 for immunotherapy of renal cell carcinoma. Clin Cancer Res 14: 6916-6923, 2008.

12. Walter S, Weinschenk T, Stenzl A, Zdrojowy R, Pluzanska A, Szczylik C, Staehler M, Brugger W, Dietrich PY, Mendrzyk R, et al: Multipeptide immune response to cancer vaccine IMA901 after single-dose cyclophosphamide associates with longer patient survival. Nat Med 18: 1254-1261, 2012.

13. Lee CS, Cragg M, Glennie M and Johnson P: Novel antibodies targeting immune regulatory checkpoints for cancer therapy. $\mathrm{Br}$ J Clin Pharmacol 76: 233-247, 2013.

14. Topalian SL, Hodi FS, Brahmer JR, Gettinger SN, Smith DC, McDermott DF, Powderly JD, Carvajal RD, Sosman JA, Atkins MB, et al: Safety, activity, and immune correlates of anti-PD-1 antibody in cancer. N Engl J Med 366: 2443-2454, 2012.

15. Mullard A: New checkpoint inhibitors ride the immunotherapy tsunami. Nat Rev Drug Discov 12: 489-492, 2013.

16. Ngiow SF, von Scheidt B, Akiba H, Yagita H, Teng MW and Smyth MJ: Anti-TIM3 antibody promotes T cell IFN- $\gamma$-mediated antitumor immunity and suppresses established tumors. Cancer Res 71: 3540-3551, 2011. 